\title{
Visual consequences of medications for multiple sclerosis: the good, the bad, the ugly, and the unknown
}

This article was published in the following Dove Press journal:

Eye and Brain

29 June 2017

Number of times this article has been viewed

\author{
Heather E Moss ${ }^{1,2}$ \\ 'Department of Ophthalmology, \\ Stanford University, Palo Alto, CA, \\ USA; '2Department of Neurology \& \\ Neuroscience, Stanford University, \\ Stanford, CA, USA
}

Correspondence: Heather E Moss Byers Eye Institute at Stanford, 2352 Watson Court, Suite 2700, Palo Alto, CA 94303, USA

Tel +l 6507249259

Fax + I 6504977936

Email hemoss@stanford.edu

\begin{abstract}
Multiple sclerosis (MS) is associated with vision changes both due to MS effects on visual pathways and due to medication effects on the visual pathways. Distinguishing the causes of vision change are critical to appropriate diagnosis and management. The incidence, presentation, and treatment of fingolimod-associated macular edema, alemtuzumab-associated thyroid orbitopathy, and progressive multifocal leukoencephalopathy in MS patients are reviewed. Evidence for beneficial effects of acute, chronic, and symptomatic MS medications on vision is presented.
\end{abstract}

Keywords: macular edema, thyroid eye disease, optic nerve, progressive multifocal leukoencephalopathy, optic neuritis

\section{Introduction}

Involvement of the afferent visual system in multiple sclerosis (MS), in particular, optic neuritis affecting the optic nerves and uveitis affecting the eye, is well described. Less appreciated are the visual consequences of therapies used for MS, the symptoms of which must be distinguished from visual consequences of MS disease to allow appropriate management.

The focus of this review is on the consequences of MS medications, both benefits and harms, as they pertain to the afferent visual pathways. The intent is neither to comprehensively review pharmacology and efficacy of MS medications nor to provide an update on MS. The interested reader is referred to an excellent review by Drs Eckstein and Bhatti for further information on these topics. ${ }^{1}$

\section{Disease-modifying agents approved for treatment of MS}

The landscape of therapies for MS is expanding (Figure 1). There are currently 16 agents (11 drug targets) approved by the US Food and Drug Administration (FDA) for the treatment of chronic MS (Table 1). Multiple additional agents are being studied in clinical trials for acute and chronic MS management, and numerous compounds are used for control of MS-related symptoms. Table 1 lists the agents currently approved by the FDA for treatment of chronic MS. The dosing route and frequency are listed to emphasize the range of dosing schedules and routes - from daily oral formulations to yearly infusions. Of particular note is alemtuzumab, which is administered in two brief cycles 12 months apart. The FDA approval dates for MS indications and nonMS indications are provided in the table to indicate the duration of experience with 


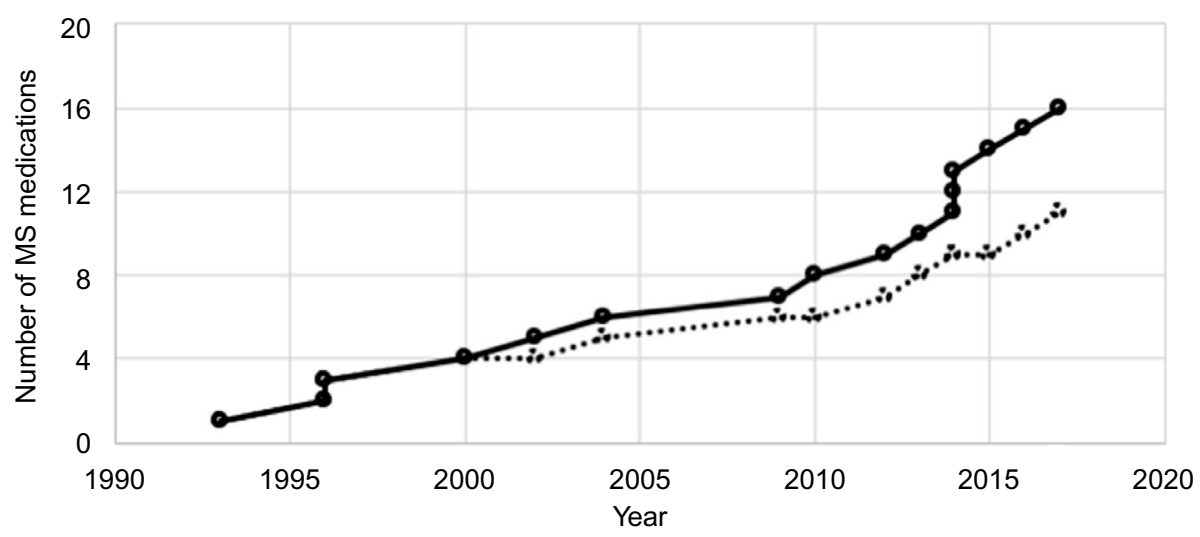

Figure I Medications approved for treatment of multiple sclerosis (MS) by the US Food and Drug Administration.

Notes: Total number of medications (solid line) reflects distinctly marketed products. Number of unique medications (dashed line) reflects number of medications with different mechanisms of action.

Table I Disease-modifying medications approved for multiple sclerosis by the FDA (as of April 20I7)

\begin{tabular}{|c|c|c|c|}
\hline $\begin{array}{l}\text { Medication name } \\
\text { (brand name) } \\
\end{array}$ & $\begin{array}{l}\text { Administration } \\
\text { route and frequency }\end{array}$ & $\begin{array}{l}\text { FDA approval } \\
\text { for MS }\end{array}$ & Prior FDA approval for other indications \\
\hline Interferon $\mathrm{Bla}$ & & & None \\
\hline (Avonex) & IM weekly & 1996 & \\
\hline (Rebif) & SC TIW & 2002 & \\
\hline Peginterferon $\mathrm{Bla}$ & SC QOW & 2014 & None \\
\hline \multicolumn{4}{|l|}{ (Plegridy) } \\
\hline Interferon BIb & SC QOD & & None \\
\hline (Betaseron) & & 1993 & \\
\hline (Extavia) & & 2009 & \\
\hline Glatiramer acetate & & & None \\
\hline \multirow[t]{2}{*}{ (Copaxone) } & SC daily & 1996 & \\
\hline & SC TIW & 2014 & \\
\hline (Glatopa - generic) & SC daily & 2015 & \\
\hline Daclizumab & SC monthly & May 2016 & 1997 as Zenapax for antiorgan transplant rejection \\
\hline \multicolumn{4}{|l|}{ (Zinbytra) } \\
\hline Mitoxantrone & IV q 3 mo & 2000 & 1987 for acute nonlymphcytic leukemia \\
\hline (Novantrone) & & & 1996 for prostate cancer \\
\hline Natalizumab & IV q $28 \mathrm{~d}$ & 2004 & None \\
\hline \multicolumn{4}{|l|}{ (Tysabri) } \\
\hline Alemtuzumab & IV daily $\times 5 \mathrm{~d}$ & 2014 & 200 I as Campath for B cell chronic lymphocytic \\
\hline (Lemtrada) & At $12 \mathrm{molV} \times 3 \mathrm{~d}$ & & leukemia \\
\hline Fingolimod & PO daily & 2010 & Studied in renal transplant 2006 \\
\hline \multicolumn{4}{|l|}{ (Gilenya) } \\
\hline Teriflunomide & PO daily & 2012 & Active metabolite of leflunomide, approved in 1998 \\
\hline (Aubagio) & & & as Arava for rheumatoid arthritis \\
\hline Dimethyl fumarate & PO BID & 2013 & None \\
\hline \multicolumn{4}{|l|}{ (Tecfidera) } \\
\hline Ocralizumab & Initially 2 IV doses 2 weeks & 2017 & None \\
\hline (Ocrevus) & apart, then IV q 6 mo & & \\
\hline
\end{tabular}

Abbreviations: BID, twice daily; d, days; FDA, US Food and Drug Administration; IM, intramuscular; IV, intravenous; mo, months; MS, multiple sclerosis; PO, per oral; q, every; QOD, every other day; QOW, every other week; SC, subcutaneous; TIW, three times weekly.

each agent (Figure 2). If interested, the reader can refer to the FDA website, ${ }^{2}$ the source of the information in Table 1, for more specific labeling information including approved indications.

\section{Evaluating the benefits and harms of therapies}

Evaluating the evidence for therapeutic harms requires a similar strategy to the evaluation of evidence with regard 


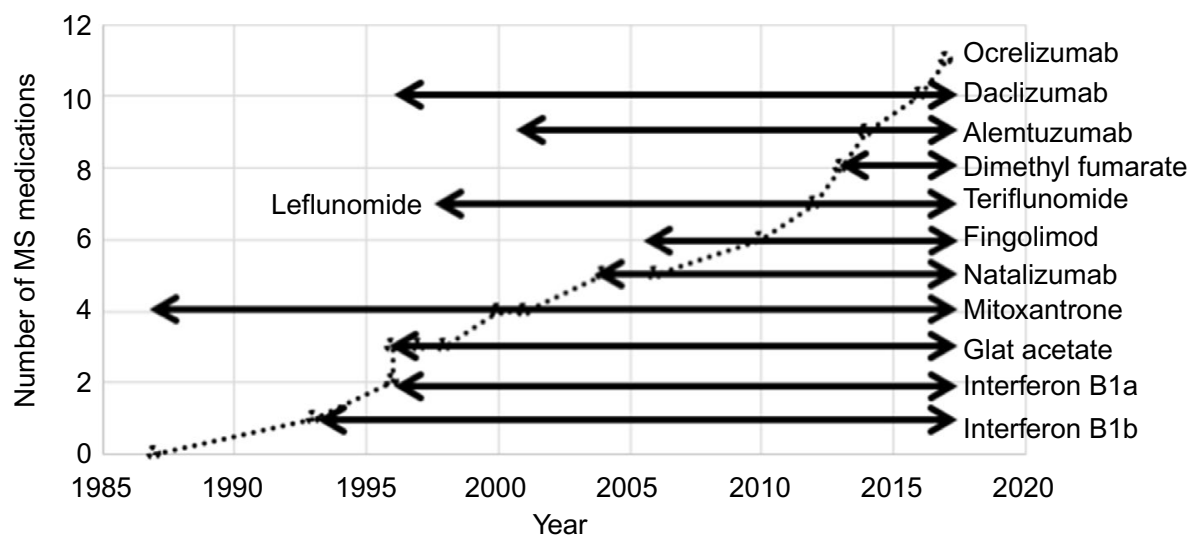

Figure 2 Duration of experience for medications approved for treatment of multiple sclerosis (MS) by the US Food and Drug Administration.

Notes: Dashed line is the number of unique medications approved as a function of year. Solid horizontal lines indicate duration of experience for each medication, with the portion to the left of the dashed diagonal line representing use for an indication other than MS and the portion to the right of the dashed diagonal line indicating use for indications including MS.

to therapeutic benefits. The Center for Evidence Based Medicine (CEBM.net) has compiled a hierarchy of likely best evidence that serves as an excellent starting point, with mechanism-based reasoning considered the lowest level of evidence and systematic review being the highest level of evidence, followed closely by randomized controlled trial. ${ }^{3}$ As with evaluation of any evidence, attention must be paid to the power of the study to detect harms, duration of the study (ie, duration of drug exposure and follow-up), and the study population. Current clinical trial designs often fail to identify rare long-term harms due to duration that is shorter than that required for the development of harms and study size under powered for the detection of rare harms.

Identifying resources from which to obtain evidence regarding harms of medications is challenging. Literature reviews using databases such as PubMed offer access to the published literature. Clinicaltrials.gov is an excellent database through which ongoing and completed trials may be identified, many of which post preliminary results. ${ }^{4}$ The FDA website includes a search function for approved drugs with links to labels and evaluations which contain information from studies used to grant the approval and also has publically available documents regarding new drug applications, with much of the included data often not available in the peer-reviewed scientific literature. ${ }^{2}$ The National Registry of Drug-induced Ocular Side Effects is an excellent resource for ophthalmic consequences of medications. ${ }^{5}$ The staff responds promptly to electronic inquires by providing published references and a copy of any relevant chapters from their book. ${ }^{6}$

Beyond case reports in the literature, multiple agencies maintain voluntary reporting systems including the FDA (the FDA Adverse Event Reporting System) ${ }^{7}$ and the National
Registry of Drug-induced Ocular Side Effects. ${ }^{5}$ FDA data files of safety reports are publically available but require an advanced level of database savviness to process and interpret. Individual safety reports can be obtained by using freedom of information requests. The National Registry of Drug-induced Ocular Side Effects also offers case-level data, given time and funds. Both of these systems are limited by reporting bias as they are constructed with voluntary reports made by treating physicians.

\section{Negative consequences of MS medications Macular edema (Fingolimod)}

Fingolimod is a nonselective sphingosine 1 phosphate (S1P) receptor modulator that limits the egress of lymphocytes from lymph nodes and also crosses the blood-brain barrier where it may have direct effects on the central nervous system. There are multiple subtypes of the $\mathrm{S} 1 \mathrm{P}$ receptor, and modulation of these is thought to be responsible for both the beneficial effects on MS and common adverse effects such as bradycardia (S1P receptors on atrial cells) or ME (S1P receptors on retinal endothelial cells altering vascular permeability). Macular effects were studied prospectively in pivotal trials of fingolimod in MS due to prior evidence of ME as a dosedependent adverse event in clinical trials of fingolimod in renal transplant. ${ }^{8,9}$

In pooled safety data from Phase II and Phase III trials of fingolimod for MS and their extension studies (2615 total patients exposed), 26 cases of ME were reported and 19 of them were confirmed. One of these cases was ultimately judged to be due to nondrug-associated branch retinal artery occlusion. Seventy-four percent were unilateral and $68 \%$ 
were symptomatic; $68 \%$ developed ME within 4 months, while $11 \%$ developed it after 12 months (mean 207 days, median 99 days). In the clinical dose group $(0.5 \mathrm{mg})$, there were five cases ( $0.3 \%$ cumulative incidence). In the higher dose group, there were 14 cases ( $1.5 \%$ cumulative incidence). Five of 26 patients (19\%) with a history of uveitis developed ME - all were in the higher dose group. ${ }^{10-12}$ Reports of atypical cases include severe bilateral ME occurring 10 days after medication initiation in a patient with diabetes ${ }^{13}$ and a single report of macular hemorrhage associated with fingolimod use. ${ }^{14}$

In addition to risk associations of dose and uveitis history, diabetes is likely associated with increased risk of fingolimod-associated ME based on the observations made during the study of fingolimod for renal transplant. In these trials, ME was seven times more common in diabetics, occurring in $28 \%$ of diabetics receiving fingolimod versus $15 \%$ of diabetics not receiving fingolimod. ${ }^{10}$ It is important to note that fingolimod doses were higher in these trials than in MS trials. Studies of fingolimod in MS excluded diabetics, and the risk of ME associated with MS dosing of fingolimod in diabetics has not been determined.

The clinical trial and FDA reports provide minimal information regarding the type of ME associated with fingolimod, though most case reports show images of cystoid changes in the central macula, consisting of medium-to-large cystoid areas, typically in the outer plexiform layer, associated with thickening of the macula. These changes are readily visible on OCT both on cross-sectional images and as an increase in macular volume. Cystoid ME from other causes such as diabetes and vitreo-macular traction can have a similar appearance on OCT. There may also be more subtle effects of fingolimod on the macula. In a study of 30 patients taking fingolimod with two groups of 30 matched MS patients not taking the drug, a small increase in macular volume $(0.025$ $\mathrm{mm}^{3}$ ) was seen in fingolimod-treated patients, but not seen in either of the two control groups..$^{15}$ The effects of fingolimod on the macula must be distinguished from microcystic changes in the inner nuclear layer of the retina that are associated with MS independent of fingolimod, with one study reporting a prevalence of $4.7 \% .{ }^{16}$ In this study, microcystic changes were not associated with increased macular volume in MS patients. It is not known if fingolimod is also associated with microcystic macular changes or if it causes progression of microcystic changes to macrocystic changes.

The FDA package insert suggests ophthalmic evaluation prior to or shortly after starting therapy and 3-4 months after starting therapy. The North American Neuro-Ophthalmology
Society (NANOS) and the American Academy of Ophthalmology Ophthalmic News and Education (AAO-ONE) Network Neuro-Ophthalmology Committee propose considering a screening evaluation for uveitis or preexisting macular disease prior to starting or within a few weeks of starting medication. They recommend an evaluation following 3-4 months of therapy and advise educating patients that the risk of ME is low, but may be increased if there is a history of uveitis or diabetes. ${ }^{17}$

For patients who develop fingolimod-associated ME, resolution typically occurs within 6 months following cessation of fingolimod, with $84 \%$ of patients in the pooled safety cohort having complete resolution..$^{910}$ Control of ME with continued fingolimod administration has been reported with frequent (every 2 hours) topical ophthalmic ketorolac and dexamethasone. ${ }^{18}$ Subtenon injections of triamcinolone were associated with resolution of ME in a single case and allowed the patient to continue on fingolimod without recurrence for 7 months. ${ }^{19}$ Intravitreal triamcinolone has been used to treat ME and allow continuation of fingolimod for at least 12 months. ${ }^{20}$

In theory, selective S1P receptor modulators should have fewer adverse effects than fingolimod, which is a nonselective receptor modulator. ${ }^{21}$ Ozanimod (RPC1063) is a selective S1P modulator being studied in Crohn disease, ulcerative colitis, and MS. In a Phase II trial, $170 \mathrm{MS}$ patients received ozanimod for 24 weeks, and there were no cases of ME. ${ }^{22}$ Siponimod (BAF312) is another selective S1P modulator that is being studied in multiple clinical trials in MS, polymyositis, and dermatomyositis. ${ }^{4}$ In a 2 -year extension study of a randomized trial where $184 \mathrm{MS}$ patients received the drug, none experienced ME. ${ }^{23}$ However, the duration of these trials and the number of patients treated are not sufficient to exclude $\mathrm{ME}$ as a rare adverse reaction at this time.

In summary, fingolimod-associated macular edema (ME) occurs in $<0.5 \%$ of patients treated for MS with an increased risk in individuals with coexisting diabetes or history of uveitis. Typical presentations are painless central vision loss (ie, reduced visual acuity) in two-third of affected individuals and asymptomatic development of cystic retinal changes on ophthalmic imaging such as optical coherence tomography (OCT) in the remaining one-third with onset 3-4 months after medication initiation. Symptoms and findings typically resolve with cessation of medication.

\section{Thyroid eye disease (alemtuzumab)}

Alemtuzumab is an anti-CD52 monoclonal antibody that has been approved for treatment of B-cell chronic lymphocytic 
leukemia since 2001 and was approved for the treatment of MS in 2014. It acts through the depletion of T and B lymphocytes. The dosing regimen is unique, consisting of five daily infusions, followed by three daily infusions after a 12-month interval. Alemtuzumab is associated with autoimmune conditions including autoimmune thyroid disease, immunemediated thrombocytopenia, and antiglomerular basement membrane disease. Though incompletely understood, it has been proposed that these associations are due to immune reconstitution in the months following treatment. ${ }^{24} \mathrm{~A}$ subset of patients with alemtuzumab-associated autoimmune thyroid dysfunction develop thyroid eye disease.

Cumulative prevalence of alemtuzumab-associated thyroid dysfunction in a Phase II trial for relapsing remitting MS was $34 \%$, with 73 of 216 patients experiencing 102 episodes. Onset was as early as 6 months and as late as 7 years following treatment, with peak incidence in year 3. About $79.5 \%$ of affected patients developed Graves' hyperthyroidism or thyroiditis with the balance developing hypothyroidism. The majority of Graves' patients were anti-TSH (thyroidstimulating hormone) receptor antibody positive at the time of diagnosis with thyroid dysfunction and a minority were anti-TPO (thyroid peroxidase) antibody positive. Younger, female patients were more likely to have thyroid dysfunction. There were no associations between the development of thyroid dysfunction and baseline characteristics of MS, MS treatment response, immune status, or immune reconstitution rates. ${ }^{25}$ Monitoring of thyroid function prior to treatment initiation and every 3 months until 48 months after last treatment course is recommended. ${ }^{24}$

In the Phase II trial for MS, 3 of 39 patients with an initial episode of thyroid dysfunction consistent with Graves' and 1 of 4 with second episode of thyroid dysfunction consistent with Graves' (following a first episode of hypothyroidism) developed significant ophthalmopathy, one of which was treated with orbital decompression. ${ }^{25}$ A case series of five patients in Phase III studies of alemtuzumab for MS includes two that had associated ophthalmopathy. In one, clinical assessment score $(\mathrm{CAS})^{26} 2$ ophthalmopathy developed 38 months after initiation of alemtuzumab and progressed to CAS 5 ophthalmopathy which was managed with IV methylprednisolone. In the other, CAS 1 ophthalmopathy was managed with artificial tears. ${ }^{27} \mathrm{~A}$ separate case report describes Graves' ophthalmopathy developing 1 year after Graves' thyroid disease was diagnosed and 3 years after alemtuzumab treatment. This patient had persistent lid retraction following thyroidectomy.$^{28}$ Due to most cases of thyroid eye disease developing after the last dose of alemtuzumab, management is similar to that of thyroid eye disease not associated with alemtuzumab.

Daclizumab is also associated with autoimmune conditions including severe autoimmune hepatitis. There are no reported cases of ophthalmic autoimmune conditions in the literature, though experience with the drug is limited as it was approved in May 2016. ${ }^{29}$

In summary, alemtuzumab-associated thyroid eye disease occurs in $<2 \%$ of patients treated for MS and is associated with alemtuzumab-associated thyroid disease, which has a 10 - to 20-fold higher incidence. Typical presentations are months to years following treatment and range from mild symptoms of eyelid retraction and ocular surface irritation to severe symptoms of restrictive orbital myopathy and compressive optic neuropathy. Management is the same as for nonalemtuzumab-associated thyroid eye disease.

\section{Opportunistic infections - progressive multifocal leukoencephalopathy (PML)}

Given the immune system modulation that is the basis of many MS therapies, it is not surprising that many are associated with opportunistic infections including PML due to JC virus infection ${ }^{31}$ and herpes virus infections. Disseminated viral infections, some of which have been fatal, have occurred in patients treated with natalizumab, ${ }^{32}$ alemtuzumab, ${ }^{24}$ and fingolimod. ${ }^{33}$ Severe infections including viral and bacterial infections have also occurred in daclizumab-treated patients. ${ }^{29}$ PML has been reported in MS patients treated with natalizumab, fingolimod, and dimethyl fumarate. ${ }^{34}$ Teriflunomide has not been associated with an increased risk of infection (serious or otherwise) compared with placebo based on a pooled safety analysis of 6400 patients on drug for a median of 670 days. ${ }^{35}$ This is unique among the newer MS disease-modifying agents.

PML became commonly recognized as a risk of MS therapy following the introduction of natalizumab in 2004 following 2 years of studies. In 2005, three cases of PML were reported in study participants and the drug was withdrawn. The following year it was reintroduced with a prescription program that included careful monitoring. Subsequent studies identified three risk factors for PML: $>24$ months of treatment, prior immunosuppressive therapy (excluding steroids, interferon B1b, B1a, and glatiramer acetate), and serum JC virus antibody detection. In 2012, the risk was estimated at 2.13:1000 overall and 11.1:1000 (95\% CI 8.3-14.5) in patients with all the three risk factors. ${ }^{36}$ At that time, no cases had been reported in any patient who was JC virus antibody negative. However, subsequent surveillance has resulted in an 
increase of the overall risk estimate to 3.96:1000 as of 2017. Cases have now been reported in patients who had had negative JCV antibody screening $<6$ months prior to diagnosis, potentially reflecting seroconversion or limited sensitivity of current screening assays. ${ }^{37}$ As with any medication, consideration of both benefits and potential harms is important in the management of MS patients using natalizumab. Longer term experience with this medication is quantifying the PML risk to allow a fair consideration.

The risk of PML is lower in MS patients treated with medications other than natalizumab. Though not seen in the clinical trials leading to its approval, PML has subsequently been reported in association with fingolimod, with nine cases reported in 150,000 treated patients $(0.056: 1000)$. It has also occurred in four cases of 224,542 patients treated with dimethyl fumarate $(0.019: 1000)$, both in association with and not associated with drug-induced lymphopenia. ${ }^{34,38} \mathrm{PML}$ has been reported in association with alemtuzumab and leflunimide (related to teriflunamide), but only in patients with diseases known to predispose to PML and not in any patients with MS including 9200 receiving alemtuzumab and 55,000 receiving teriflunamide. ${ }^{34}$ PML has not yet been reported in association with daclizumab treatment, though duration and volume of experience are minimal at this time (1516 treated patients).

Diagnosis of PML is based on clinical suspicion, imaging findings, testing for JC virus in the cerebrospinal fluid, and sometimes brain biopsy. Typical MRI findings are T2 hyperintense and T1 hypointense lesions affecting the white matter including subcortical U-fibers, though posterior fossa involvement is not uncommon. Enhancement is variable. ${ }^{39}$

Though there are no proven treatments, several approaches have been associated with improved outcomes at the case series level including cessation of drug and plasma exchange in natalizumab-associated PML. ${ }^{40}$ Multiple other pharmacologic agents have been associated with improvement in individual patients. A challenge in PML management is that some therapies such as cessation of and removal of immunomodulatory therapy can provoke immune reconstitution inflammatory syndrome (IRIS), which can also cause central nervous system injury. ${ }^{41}$ Clinical deterioration associated with increase in gadolinium contrast enhancement on MRI suggests IRIS, which is typically managed using glucocorticoids.

In summary, PML is a life-threatening infection due to John Cunningham (JC) virus that presents with focal neurological deficits and often seizures. Visual presentation of PML is common, with homonymous visual field loss being found in $\sim 20 \%-25 \%$ of cases.30 Other visual symptoms include higher order visual dysfunction, nystagmus, and brainstem syndromes. Diagnosis is based on neuroimaging and spinal fluid testing. Therapies include drug cessation, plasma exchange, and other immune modulation.

\section{Positive consequence of MS medications \\ Lack of visual function decline in chronic MS}

Some efficacy trials for MS medications have included visual outcomes. Two Phase III trials of natalizumab included low-contrast visual acuity (LCVA) as a tertiary endpoint. ${ }^{42}$ In these, $100 \%, 2.5 \%$, and $1.25 \%$ binocular contrast visual acuity (VA) measurements were performed every 12 weeks for 10 study visits. $Z$-scores for change from baseline demonstrated a decline of $2.5 \%$ and $1.25 \%$ LCVA in 315 patients receiving placebo and stability of vision in 627 natalizumab-treated patients, with statistical difference between the two groups seen at the first 12-week visit and increasing in magnitude at future visits. Prevalence of sustained worsening of $100 \%$ contrast VA was low and similar between treatment groups. There was a lower cumulative probability of sustained $1.25 \%$ contrast VA worsening in natalizumab-treated patients over 120 months.

In a Phase II trial of alemtuzumab versus interferon $\beta$ - $1 \mathrm{a}$ (IFNB-1a) for treatment-naïve patients with relapsingremitting $\mathrm{MS}$, contrast sensitivity was evaluated as an exploratory efficacy outcome in 273 of 324 patients. This was performed in each eye using Pelli-Robson charts. Sustained improvement was more likely to occur and sustained worsening less likely to occur in alemtuzumab versus IFNB1a-treated patients, with both groups showing small though statistically significant improvements in contrast sensitivity over 36 months. ${ }^{43}$ It is not known if these results can be extrapolated to other medications.

\section{Improved visual function following optic neuritis}

Opicinumab is a monoclonal antibody against LINGO-1, which is involved in the regulation of myelination, and has been studied in acute optic neuritis and MS. Safety and tolerability data in 72 healthy individuals and 47 MS patients were published in $2014 .{ }^{44} \mathrm{~A}$ treatment course of 32 weeks was studied in a randomized, placebo controlled trial of 82 patients with acute optic neuritis. All subjects also received standard-of-care treatment consisting of pulse IV methylprednisolone. Visual evoked potential (VEP) latency was shorter in treated patients, suggesting a remyelinating effect. ${ }^{45}$ 
Neuroprotective strategies are being actively investigated to decrease axonal loss during and following optic neuritis episodes. A recently completed Phase II trial of 86 patients suggested that phenytoin administered for 3 months following optic neuritis was associated with less retinal nerve fiber layer (RNFL) loss, an imaging measure of optic nerve integrity, in comparison with placebo-treated patients. ${ }^{46}$ An open-label trial of high-dose biotin in patients with primary or secondary progressive MS included four with prominent optic nerve involvement, all of whom had visual acuity improvement over 3 months. ${ }^{47}$ Two of the patients had VEPs recorded and demonstrated improvement. A recent trial of amiloride in acute optic neuritis ${ }^{48}$ did not show benefit (Presented at European Committee for Treatment and Research in Multiple Sclerosis Meeting; M. Craner, personal communication).

4-Aminopyridine(4-AP), the immediate-release form of dalfampridine, is a potassium channel blocker that improves conduction in demyelinated neurons. Dalfampridine was studied for, and has FDA approval for, the treatment of slow gait in MS. 4-AP was studied in a randomized study of 22 patients with chronic optic neuropathy defined by RNFL thinning. LCVA, RNFL, and VEP were studied as outcome measures with 14 patients included in the final analysis (after exclusion for MS relapses during the study and nonadherence). Eight of 28 eyes treated with 4-AP showed improvement in LCVA compared with three of 28 placebo eyes, though there was no group difference. VEP latency improved in the 4-AP group but declined in the placebo group, and this beneficial effect was more pronounced in eyes with less RNFL thinning (ie, less optic nerve axonal loss). ${ }^{49} 4$-AP has also shown beneficial effects on nystagmus, ${ }^{50}$ as have other agents. Dalfampridine, the commercially available formulation, has not been formally studied for vision or nystagmus outcomes.

\section{Delay of progression to MS following optic neuritis}

Multiple trials in the current century have evaluated IFNB$1 \mathrm{~b}$, glatiramer acetate, and cladrabine in patients with clinically isolated syndromes, including optic neuritis, and brain lesions on MRI (typically two or more). All showed a modest benefit of treatment in comparison with placebo on cumulative incidence of second clinical demyelinating event and development of new MRI lesions indicative of diagnosis with clinically definite MS. Revision of MS diagnostic criteria in 2010, to allow diagnosis of MS following clinically isolated syndrome with concurrent radiographic demonstration of dissemination in time and space, has complicated interpretation of some of these studies since many included patients with enhancing brain lesions. ${ }^{51}$ Due to the length of the trials, generally $2-3$ years, long-term benefits including effects on disability have not been studied. Practice regarding treatment of optic neuritis patients with nonenhancing brain lesions on MRI varies throughout the world. ${ }^{52}$

Only one of the newer MS therapies, teriflunomide, has been studied with regard to delaying onset of clinically definite MS in patients with clinically isolated syndrome and two or more T2 lesions on brain MRI. Similar to other agents studied, subjects receiving teriflunomide had both decreased progression to clinically definite MS and fewer new T2 lesions on MRI over 2 years. However, development of disability was no different between groups. ${ }^{53}$

\section{Conclusion}

In trials of disease-modifying MS medications that have included visual outcomes, treatment benefit on visual function has been demonstrated. There are candidates in the pipeline and on the market to improve visual outcomes following acute optic neuritis and to improve optic nerve function in patients with established optic neuropathy. Two recently approved disease-modifying MS drugs have specific neuro-ophthalmic risks. Fingolimod is associated with a low risk of ME that responds well to treatment discontinuation. Alemtuzumab is associated with autoimmune thyroid dysfunction and thyroid orbitopathy in a proportion of those affected. Many of the newer disease-modifying MS drugs have powerful immune effects and are associated with potentially life-threatening infections including PML and herpes virus infections. However, absence of evidence is not evidence of absence with regard to both benefit and harm. Clinical experience in terms of numbers of patients treated and longer durations of treatment are necessary to determine the incidence and extent of many harmful consequences.

\section{Acknowledgment}

This work was supported by K23 EY 024345 (National Eye Institute) and an unrestricted grant from Research to Prevent Blindness to Stanford Department of Ophthalmology.

\section{Disclosure}

The author reports no conflicts of interest in this work.

\section{References}

1. Eckstein C, Bhatti MT. Currently approved and emerging oral therapies in multiple sclerosis: An update for the ophthalmologist. Surv Ophthalmol. 2016;61(3):318-332.

2. Drugs@FDA: FDA Approved Drug Products. 2016; Available from: https://www.accessdata.fda.gov/scripts/cder/drugsatfda/. Accessed September 6, 2016. 
3. Group OLoEW. The Oxford 2011 Levels of Evidence. Available from: http://cebm.net/index.aspx?o=5653. Accessed August 6, 2016.

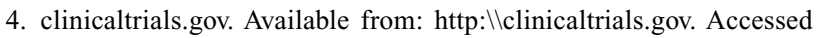
September 7, 2016.

5. National Registry of Drug-Induced Ocular Side Effects. Available from: http: \leyedrugregistry.com. Accessed September 6, 2016.

6. Fraunfelder F, Fraunfelder F, Chambers W. Drug-Induced Ocular Side Effects. 7th ed. Elsevier; 2014.

7. FDA Adverse Events Resporting System. Available from: http://www. fda.gov/Drugs/GuidanceComplianceRegulatoryInformation/Surveillance/AdverseDrugEffects/. Accessed September 7, 2016.

8. Salvadori M, Budde K, Charpentier B, et al; FTY720 0124 Study Group. FTY720 versus MMF with cyclosporine in de novo renal transplantation: a 1-year, randomized controlled trial in Europe and Australasia. Am J Transplant. 2006;6(12):2912-2921.

9. Jain N, Bhatti MT. Fingolimod-associated macular edema: incidence, detection, and management. Neurology. 2012;78(9):672-680.

10. Zarbin MA, Jampol LM, Jager RD, et al. Ophthalmic evaluations in clinical studies of fingolimod (FTY720) in multiple sclerosis. Ophthalmology. 2013;120(7):1432-1439.

11. Research FaDACfDEa. Fingolimod (NDA 22-527) Background Package. Peripheral and Central Nervous System Advisory Committee Meeting; 2010. Available from: http://www.fda.gov/downloads/AdvisoryCommittees/CommitteesMeetingMaterials/Drugs/PeripheralandCentralNervousSystemDrugsAdvisoryCommittee/UCM214670.pdf. Accessed September 8, 2016.

12. Research FaDACfDEa. Fingolimod (NDA 22-527) Presentation. Peripheral and Central Nervous System Advisory Committee Meeting; 2010. Available from: http://www.fda.gov/downloads/AdvisoryCommittees/ CommitteesMeetingMaterials/Drugs/PeripheralandCentralNervousSystemDrugsAdvisoryCommittee/UCM216553.pdf. Accessed September 8, 2016.

13. Coppes OJ, Gutierrez I, Reder AT, Ksiazek S, Bernard J. Severe early bilateral macular edema following fingolimod therapy. Mult Scler Relat Disord. 2013;2(3):256-258.

14. Bhatti MT, Freedman SM, Mahmoud TH. Fingolimod therapy and macular hemorrhage. J Neuroophthalmol. 2013;33(4):370-372.

15. Nolan R, Gelfand JM, Green AJ. Fingolimod treatment in multiple sclerosis leads to increased macular volume. Neurology. 2013;80(2): 139-144.

16. Gelfand JM, Nolan R, Schwartz DM, Graves J, Green AJ. Microcystic macular oedema in multiple sclerosis is associated with disease severity. Brain. 2012;135(Pt 6): 1786-1793.

17. Lee AG. Ophthalmic screening recommendation for new MS drug: a report from the North American Neuro-Ophthalmology Society (NANOS) and the AAO Ophthalmic News and Education (ONE) Network Neuro-Ophthalmology Committee. Am Academy of Ophthalmology ONE Network; 2011. Available from: http://www.aao.org/ editors-choice/ophthalmic-screening-recommendation-new-ms-drug. Accessed September 8, 2016.

18. Chui J, Herkes GK, Chang A. Management of fingolimod-associated macular edema. JAMA Ophthalmol. 2013;131(5):694-696.

19. Minuk A, Belliveau MJ, Almeida DR, Dorrepaal SJ, Gale JS. Fingolimod-associated macular edema: resolution by sub-tenon injection of triamcinolone with continued fingolimod use. JAMA Ophthalmol. 2013;131(6):802-804.

20. Thoo S, Cugati S, Lee A, Chen C. Successful treatment of fingolimodassociated macular edema with intravitreal triamcinolone with continued fingolimod use. Mult Scler. 2015;21(2):249-251.

21. Sorensen PS. Ozanimod: a better or just another S1P receptor modulator? Lancet Neurol. 2016;15(4):345-347.

22. Cohen JA, Arnold DL, Comi G, et al. Safety and efficacy of the selective sphingosine 1-phosphate receptor modulator ozanimod in relapsing multiple sclerosis (RADIANCE): a randomised, placebo-controlled, phase 2 trial. Lancet Neurol. 2016;15(4):373-381.
23. Kappos L, Li DK, Stuve O, et al. Safety and efficacy of siponimod (BAF312) in patients with relapsing-remitting multiple sclerosis: dose-blinded, randomized extension of the phase 2 BOLD study. JAMA Neurol. 2016;73(9):1089-1098.

24. Willis MD, Robertson NP. Alemtuzumab for multiple sclerosis. Curr Neurol Neurosci Rep. 2016;16(9):84.

25. Daniels GH, Vladic A, Brinar V, et al. Alemtuzumab-related thyroid dysfunction in a phase 2 trial of patients with relapsing-remitting multiple sclerosis. J Clin Endocrinol Metab. 2014;99(1):80-89.

26. Wiersinga WM, Perros P, Kahaly GJ, et al. Clinical assessment of patients with Graves' orbitopathy: the European Group on Graves' Orbitopathy recommendations to generalists, specialists and clinical researchers. Eur J Endocrinol. 2006;155(3):387-389.

27. Tsourdi E, Gruber M, Rauner M, Blankenburg J, Ziemssen T, Hofbauer LC. Graves' disease after treatment with alemtuzumab for multiple sclerosis. Hormones (Athens). 2015;14(1):148-153.

28. Trinh T, Haridas AS, Sullivan TJ. Ocular findings in alemtuzumab (Campath-1H)-induced thyroid eye disease. Ophthal Plast Reconstr Surg. 2016;32(6):e128-e129.

29. Dunn B. FDA Center for Drug Evaluation and Research Summary Review: application number 761029Orig1s000 (Zynbryta/daclizumb); 2016. Available from: http://www.accessdata.fda.gov/drugsatfda_docs/ nda/2016/761029Orig1s000SumR.pdf. Accessed September 8, 2016.

30. Sudhakar P, Bachman DM, Mark AS, Berger JR, Kedar S. Progressive multifocal leukoencephalopathy: recent advances and a neuro-ophthalmological review. J Neuroophthalmol. 2015;35(3): 296-305.

31. Faulkner M. Risk of progressive multifocal leukoencephalopathy in patients with multiple sclerosis. Exp Opin Drug Saf. 2015;14(11): 1737-1748.

32. Fine AJ, Sorbello A, Kortepeter C, Scarazzini L. Central nervous system herpes simplex and varicella zoster virus infections in natalizumabtreated patients. Clin Infect Dis. 2013;57(6):849-852.

33. Khatri BO. Fingolimod in the treatment of relapsing-remitting multiple sclerosis: long-term experience and an update on the clinical evidence. Ther Adv Neurol Disord. 2016;9(2):130-147.

34. Berger JR. Classifying PML risk with disease modifying therapies. Mult Scler Relat Disord. 2017;12:59-63.

35. Papadopoulou A, Kappos L, Sprenger T. Safety of teriflunomide for the management of relapsing-remitting multiple sclerosis. Exp Opin Drug Saf. 2015;14(5):749-759.

36. Bloomgren G, Richman S, Hotermans C, et al. Risk of natalizumabassociated progressive multifocal leukoencephalopathy. $N$ Engl J Med. 2012;366(20):1870-1880.

37. Schwab N, Schneider-Hohendorf T, Melzer N, Cutter G, Wiendl H. Natalizumab-associated PML: challenges with incidence, resulting risk, and risk stratification. Neurology. 2017;88(12):1197-1205.

38. Ermis U, Weis J, Schulz JB. PML in a patient treated with fumaric acid. N Engl J Med. 2013;368(17):1657-1658.

39. Berger JR, Aksamit AJ, Clifford DB, et al. PML diagnostic criteria: consensus statement from the AAN neuroinfectious disease section. Neurology. 2013;80(15):1430-1438.

40. Saylor D, Venkatesan A. Progressive multifocal leukoencephalopathy in HIV-uninfected individuals. Curr Infect Dis Rep. 2016;18(11):33.

41. N'Gbo N'gbo Ikazabo R, Mostosi C, Quivron B, Delberghe X, El Hafsi $\mathrm{K}$, Lysandropoulos AP. Immune-reconstitution inflammatory syndrome in multiple sclerosis patients treated with natalizumab: a series of 4 cases. Clin Ther. 2016;38(3):670-675.

42. Balcer LJ, Galetta SL, Calabresi PA, et al. Natalizumab reduces visual loss in patients with relapsing multiple sclerosis. Neurology. 2007;68(16):1299-1304.

43. Graves J, Galetta SL, Palmer J, et al. Alemtuzumab improves contrast sensitivity in patients with relapsing-remitting multiple sclerosis. Mult Scler. 2013;19(10):1302-1309. 
44. Tran JQ, Rana J, Barkhof F, et al. Randomized phase I trials of the safety/tolerability of anti-LINGO-1 monoclonal antibody BIIB033. Neurol Neuroimmunol Neuroinflamm. 2014;1(2):e18.

45. Cadavid D, Balcer L, Galetta S, et al. Safety and efficacy of opicinumab in acute optic neuritis (RENEW): a randomised, placebo-controlled, phase 2 trial. Lancet Neurol. 2017;16(3):189-199.

46. Raftopoulos R, Hickman SJ, Toosy A, et al. Phenytoin for neuroprotection in patients with acute optic neuritis: a randomised, placebocontrolled, phase 2 trial. Lancet Neurol. 2016;15(3):259-269.

47. Sedel F, Papeix C, Bellanger A, et al. High doses of biotin in chronic progressive multiple sclerosis: a pilot study. Mult Scler Relat Disord. 2015;4(2):159-169.

48. McKee JB, Elston J, Evangelou N, et al. Amiloride Clinical Trial In Optic Neuritis (ACTION) protocol: a randomised, double blind, placebo controlled trial. BMJ open. 2015;5(11):e009200.
49. Horton L, Conger A, Conger D, et al. Effect of 4-aminopyridine on vision in multiple sclerosis patients with optic neuropathy. Neurology. 2013;80(20): 1862-1866.

50. Claassen J, Spiegel R, Kalla R, et al. A randomised double-blind, cross-over trial of 4-aminopyridine for downbeat nystagmus-effects on slowphase eye velocity, postural stability, locomotion and symptoms. J Neurol, Neurosurg Psychiatry. 2013;84(12):1392-1399.

51. Montalban X, Sastre-Garriga J. Diagnosis and trials of clinically isolated syndrome. Lancet Neurol. 2014;13(10):962-963.

52. Hickman SJ, Dalton CM, Miller DH, Plant GT. Management of acute optic neuritis. Lancet. 2002;360(9349):1953-1962.

53. Miller AE, Wolinsky JS, Kappos L, et al. Oral teriflunomide for patients with a first clinical episode suggestive of multiple sclerosis (TOPIC): a randomised, double-blind, placebo-controlled, phase 3 trial. Lancet Neurol. 2014;13(10):977-986.
Eye and Brain

\section{Publish your work in this journal}

Eye and Brain is an international, peer-reviewed, open access journal focusing on clinical and experimental research in the field of neuroophthalmology. All aspects of patient care are addressed within the journal as well as basic research. Papers covering original research, basic science, clinical and epidemiological studies, reviews and evaluations,

Submit your manuscript here: https://www.dovepress.com/eye-and-brain-journal

\section{Dovepress}

guidelines, expert opinion and commentary, case reports and extended reports are welcome. The manuscript management system is completely online and includes a very quick and fair peer-review system, which is all easy to use. Visit http://www.dovepress.com/testimonials.php to read real quotes from published authors. 